MATHEMATICS OF COMPUTATION

Volume 79, Number 271, July 2010, Pages 1709-1725

S 0025-5718(09)02307-2

Article electronically published on November 19, 2009

\title{
ON THE EVALUATION OF MATSUBARA SUMS
}

\author{
OLIVIER ESPINOSA
}

Abstract. Given a connected (multi)graph $G$, consisting of $V$ vertices and $I$ lines, we consider a class of multidimensional sums of the general form

$$
S_{G}:=\sum_{n_{1}=-\infty}^{\infty} \sum_{n_{2}=-\infty}^{\infty} \cdots \sum_{n_{I}=-\infty}^{\infty} \frac{\delta_{G}\left(n_{1}, n_{2}, \ldots, n_{I} ;\left\{N_{v}\right\}\right)}{\left(n_{1}^{2}+q_{1}^{2}\right)\left(n_{2}^{2}+q_{2}^{2}\right) \cdots\left(n_{I}^{2}+q_{I}^{2}\right)},
$$

where the variables $q_{i}(i=1, \ldots, I)$ are real and positive and the variables $N_{v}$ $(v=1, \ldots, V)$ are integer-valued. $\delta_{G}\left(n_{1}, n_{2}, \ldots, n_{I} ;\left\{N_{v}\right\}\right)$ is a function valued in $\{0,1\}$ which imposes a series of linear constraints among the summation variables $n_{i}$, determined by the topology of the graph $G$.

We prove that these sums, which we call Matsubara sums, can be explicitly evaluated by applying a $G$-dependent linear operator $\hat{\mathcal{O}}_{G}^{\prime}$ to the evaluation of the integral obtained from $S_{G}$ by replacing the discrete variables $n_{i}$ by continuous real variables $x_{i}$ and replacing the sums by integrals.

\section{INTRODUCTION}

Infinite series are ubiquitous in mathematics. In particular, both elementary and special functions are either defined in terms of an infinite series or have series representations of one sort or another. For example, the Hurwitz zeta function is defined by the series

$$
\zeta(z, q):=\sum_{n=0}^{\infty} \frac{1}{(n+q)^{z}},
$$

for $z \in \mathbb{C}, \operatorname{Re} z>1$ and $q \in \mathbb{C}, q \neq 0,-1,-2, \ldots$ The Riemann zeta function is a special case of the Hurwitz zeta function, $\zeta(z)=\zeta(z, 1)$.

Conversely, confronted with an infinite series, it is always a legitimate pursuit to try to evaluate it in terms of known elementary or special functions. Sometimes, as an intermediate step, the evaluation of infinite series can be reduced to the evaluation of an integral, which may or may not have a closed form evaluation. Consider, for instance, Plana's summation formula [1,

$$
\sum_{n=0}^{\infty} f(n)=\frac{1}{2} f(0)+\int_{0}^{\infty} f(x) d x+i \int_{0}^{\infty} \frac{f(i t)-f(-i t)}{e^{2 \pi t}-1} d t
$$

valid under certain restrictive growth conditions for the function $f$ in the complex domain.

Received by the editor March 13, 2009 and, in revised form, May 19, 2009.

2000 Mathematics Subject Classification. Primary 33-XX; Secondary 33E20, 33F99.

(C)2009 American Mathematical Society Reverts to public domain 28 years from publication 
When applied to the series (1.1), Plana's formula leads to Hermite's representation [2],

$$
\zeta(z, q)=\frac{1}{2} q^{-z}+\frac{1}{z-1} q^{1-z}+2 \int_{0}^{\infty} \frac{\sin \left(z \tan ^{-1}(t / q)\right)}{\left(q^{2}+t^{2}\right)^{z / 2}\left(e^{2 \pi t}-1\right)} d t,
$$

for $\operatorname{Re} q>0$. This representation actually provides a meromorphic extension of $\zeta(z, q)$ to the whole complex $z$-plane.

A formula similar in spirit to Plana's summation formula can be obtained by use of contour integration and the residue theorem,

$$
\sum_{n=-\infty}^{\infty} f(n)=\frac{1}{2 \pi i} \oint_{\downarrow \uparrow} 2 \pi \mathrm{n}_{\mathrm{b}}(z) f(-i z) d z,
$$

provided the function $f(z)$ does not have poles on the imaginary axis. The contour denoted above by $\downarrow$ runs parallel to the imaginary axis, upwards from the right and downwards from the left, encircling counterclockwise all the poles (located at $z=n i, n \in \mathbb{Z})$ of the kernel $\mathrm{n}_{\mathrm{b}}(z)$, defined as

$$
\mathrm{n}_{\mathrm{b}}(z)=\frac{1}{e^{2 \pi z}-1}=\frac{1}{2}(\operatorname{coth} \pi z-1) .
$$

If the function $f(-i z)$ is such that its integral along a circular contour at infinity vanishes, then we can evaluate the contour integral in (1.4) by splitting the original contour $\downarrow$ into two closed clockwise contours, one on each side of the imaginary axis and enclosing all the poles of $f(-i z)$. As a simple example, consider the evaluation of the sum

$$
\sum_{n=-\infty}^{\infty} \frac{1}{n^{2}+q^{2}}
$$

where $q$ is a positive real variable. In this case we have the evaluation

$$
\begin{aligned}
\frac{1}{2 \pi i} \oint_{\Downarrow \uparrow} 2 \pi \mathrm{n}_{\mathrm{b}}(z) \frac{1}{-z^{2}+q^{2}} d z & =2 \pi \sum_{z= \pm q} \operatorname{Res}_{z}\left[\mathrm{n}_{\mathrm{b}}(z) \frac{1}{z^{2}-q^{2}}\right] \\
& =\frac{2 \pi}{2 q}\left[\mathrm{n}_{\mathrm{b}}(q)-\mathrm{n}_{\mathrm{b}}(-q)\right] .
\end{aligned}
$$

The identity

$$
\mathrm{n}_{\mathrm{b}}(z)+\mathrm{n}_{\mathrm{b}}(-z)+1=0
$$

can be used to obtain the known result,

$$
\sum_{n=-\infty}^{\infty} \frac{1}{n^{2}+q^{2}}=\frac{\pi \operatorname{coth} \pi q}{q}
$$

In this paper we consider the evaluation of a class of sums, $\mathfrak{M}$, that generalizes the simple sum (1.6). Each of the sums in $\mathfrak{M}$ is defined in terms of a particular kind of connected graph, in a way that we make explicit in the next section.

In principle, the sums in $\mathfrak{M}$, called Matsubara sums, can be evaluated by the repeated direct application of the contour integration formula (1.4), on a case-by-case basis. However, using an algebraic identity, M. Gaudin 8 has been able to obtain a closed form evaluation of any Matsubara sum as a sum of terms corresponding to the trees of the corresponding graph. This is reviewed in section 4. 
Starting from Gaudin's result, we prove that any Matsubara sum can be alternatively evaluated by applying a linear operator to the evaluation of an integral associated with the sum. Although this integral can also be computed using Gaudin's method, it is usually the case that the direct computation of the integral can be done in a straightforward manner by other means, including symbolic manipulation programs such as Mathematica or Maple. In this case the evaluation of the corresponding sum will be notably simplified.

The general results presented in this paper are a by-product of the study of general properties of Feynman graphs in the so-called Euclidean or imaginarytime formalism of finite-temperature quantum field theory. In this formalism the evaluation of each Feynman graph requires the computation of a sum of Matsubara type. The existence of the linear operator referred to above was first conjectured from the analysis of two general classes of Feynman graphs [6] and then established in full generality [7. The aim of this paper is to present the relevant mathematical results for a readership of non-(particle) physicists. Accordingly, all references to physical quantities have been removed.

Before defining our class $\mathfrak{M}$ in full generality and presenting the general theorems, we will illustrate our main results for the simplest non-trivial sum in $\mathfrak{M}$, which we will call $S_{G_{2}}$ :

$$
S_{G_{2}}:=\sum_{n_{1}=-\infty}^{\infty} \sum_{n_{2}=-\infty}^{\infty} \frac{\delta_{n_{1}+n_{2}-N, 0}}{\left(n_{1}^{2}+q_{1}^{2}\right)\left(n_{2}^{2}+q_{2}^{2}\right)}=\sum_{n=-\infty}^{\infty} \frac{1}{\left(n^{2}+q_{1}^{2}\right)\left((N-n)^{2}+q_{2}^{2}\right)} .
$$

Here $N \in \mathbb{Z}$ and $q_{1}>0, q_{2}>0$ are real variables. The Kronecker delta symbol is defined for $n, m \in \mathbb{Z}$ by

$$
\delta_{n, m}= \begin{cases}1 & \text { if } n=m \\ 0 & \text { otherwise }\end{cases}
$$

A standard evaluation, by the method of residues (1.4) for example, yields

$$
\begin{aligned}
\sum_{n=-\infty}^{\infty} \frac{1}{\left(n^{2}+q_{1}^{2}\right)\left((N-n)^{2}+q_{2}^{2}\right)}=\frac{2 \pi}{2 q_{1} 2 q_{2}}\left[\frac{1+\mathrm{n}_{\mathrm{b}}\left(q_{1}\right)+\mathrm{n}_{\mathrm{b}}\left(q_{2}\right)}{N i+q_{1}+q_{2}}\right. \\
\left.+\frac{\mathrm{n}_{\mathrm{b}}\left(q_{1}\right)-\mathrm{n}_{\mathrm{b}}\left(q_{2}\right)}{N i-q_{1}+q_{2}}-\frac{\mathrm{n}_{\mathrm{b}}\left(q_{1}\right)-\mathrm{n}_{\mathrm{b}}\left(q_{2}\right)}{N i+q_{1}-q_{2}}-\frac{1+\mathrm{n}_{\mathrm{b}}\left(q_{1}\right)+\mathrm{n}_{\mathrm{b}}\left(q_{2}\right)}{N i-q_{1}-q_{2}}\right],
\end{aligned}
$$

where $\mathrm{n}_{\mathrm{b}}(q)$ is the kernel defined in (1.5).

We note that, although written using the imaginary unit $i=\sqrt{-1}$, the result is clearly real. In the form just written, it becomes easier to recognize the general structure of the results we shall present below.

Consider the integral obtained by replacing, in the LHS of (1.11), the sum over the discrete variable $n$ by an integral over a continuous variable $x$ :

$$
\begin{aligned}
I_{G_{2}}:=\int_{-\infty}^{\infty} \frac{d x}{\left(x^{2}+q_{1}^{2}\right)\left((N-x)^{2}+q_{2}^{2}\right)} & =\frac{\pi\left(q_{1}+q_{2}\right)}{q_{1} q_{2}\left(N^{2}+\left(q_{1}+q_{2}\right)^{2}\right)} \\
& =\frac{2 \pi}{2 q_{1} 2 q_{2}}\left[\frac{1}{N i+q_{1}+q_{2}}-\frac{1}{N i-q_{1}-q_{2}}\right] .
\end{aligned}
$$


It is clear from these explicit evaluations that for this simple case there is a simple formal relation between the sum $S_{G_{2}}$ and the integral $I_{G_{2}}$. To formulate this relation we introduce reflection operators $\hat{R}_{i} \equiv \hat{R}\left(q_{i}\right)$, acting on the space of functions of the variables $q_{j}$,

$$
\hat{R}\left(q_{i}\right) f\left(q_{1}, \ldots, q_{i}, \ldots\right)=f\left(q_{1}, \ldots,-q_{i}, \ldots\right) .
$$

For instance, we have

$$
\hat{R}\left(q_{1}\right)\left[\frac{2 \pi}{2 q_{1} 2 q_{2}} \frac{1}{N i-q_{1}-q_{2}}\right]=-\frac{2 \pi}{2 q_{1} 2 q_{2}} \frac{1}{N i+q_{1}-q_{2}}
$$

and

$$
\hat{R}\left(q_{2}\right)\left[\frac{2 \pi}{2 q_{1} 2 q_{2}} \frac{1}{N i+q_{1}+q_{2}}\right]=-\frac{2 \pi}{2 q_{1} 2 q_{2}} \frac{1}{N i+q_{1}-q_{2}} .
$$

In terms of the reflection operator (1.13) we have:

$$
S_{G_{2}}\left(N, q_{1}, q_{2}\right)=\left[1+\mathrm{n}_{\mathrm{b}}\left(q_{1}\right)\left(1-\hat{R}\left(q_{1}\right)\right)+\mathrm{n}_{\mathrm{b}}\left(q_{2}\right)\left(1-\hat{R}\left(q_{2}\right)\right)\right] I_{G_{2}}\left(N, q_{1}, q_{2}\right) .
$$

For instance, the term

$$
-\frac{2 \pi}{2 q_{1} 2 q_{2}} \frac{\mathrm{n}_{\mathrm{b}}\left(q_{1}\right)}{N i+q_{1}-q_{2}}
$$

in (1.11) is generated as

$$
-\frac{2 \pi}{2 q_{1} 2 q_{2}} \frac{\mathrm{n}_{\mathrm{b}}\left(q_{1}\right)}{N i+q_{1}-q_{2}}=-\mathrm{n}_{\mathrm{b}}\left(q_{1}\right) \hat{R}\left(q_{1}\right)\left[-\frac{2 \pi}{2 q_{1} 2 q_{2}} \frac{1}{N i-q_{1}-q_{2}}\right] .
$$

It is straightforward to show that the operator $\left(1-\hat{R}_{1}\right)\left(1-\hat{R}_{2}\right)$ annihilates the integral $I_{G_{2}}$ evaluated in (1.12), that is:

$$
\left(1-\hat{R}_{1}\right)\left(1-\hat{R}_{2}\right) I_{G_{2}} \equiv 0,
$$

where we have abbreviated $\hat{R}_{i}:=\hat{R}\left(q_{i}\right)$. Therefore, the operator in (1.14) that generates the sum $S_{G_{2}}$ from the corresponding integral $I_{G_{2}}$ can be written in the multiplicative form,

$$
\hat{\mathcal{O}}_{G_{2}}=\prod_{i=1}^{2}\left[1+\mathrm{n}_{\mathrm{b} i}\left(1-\hat{R}_{i}\right)\right]
$$

where $\mathrm{n}_{\mathrm{b} i}:=\mathrm{n}_{\mathrm{b}}\left(q_{i}\right)$.

In this paper we shall prove that all the sums in the class $\mathfrak{M}$, to be defined in the next section, satisfy a property similar to (1.14), with an operator of the type (1.16).

Note 1.1. It is important to notice that whereas the evaluation (1.12) is still valid if the variable $N$ is extended to the real or complex domains, the same does not happen in the case of (1.11). In the latter case one finds, for $N \in \mathbb{C}$,

$$
\begin{aligned}
& \text { 17) } \sum_{n=-\infty}^{\infty} \frac{1}{\left(n^{2}+q_{1}^{2}\right)\left((N-n)^{2}+q_{2}^{2}\right)}=\frac{2 \pi}{2 q_{1} 2 q_{2}}\left[\frac{1+\mathrm{n}_{\mathrm{b}}\left(q_{1}\right)+\mathrm{n}_{\mathrm{b}}\left(q_{2}+N i\right)}{N i+q_{1}+q_{2}}\right. \\
& \left.+\frac{\mathrm{n}_{\mathrm{b}}\left(q_{1}\right)-\mathrm{n}_{\mathrm{b}}\left(q_{2}+N i\right)}{N i-q_{1}+q_{2}}-\frac{\mathrm{n}_{\mathrm{b}}\left(q_{1}\right)-\mathrm{n}_{\mathrm{b}}\left(q_{2}-N i\right)}{N i+q_{1}-q_{2}}-\frac{1+\mathrm{n}_{\mathrm{b}}\left(q_{1}\right)+\mathrm{n}_{\mathrm{b}}\left(q_{2}-N i\right)}{N i-q_{1}-q_{2}}\right] .
\end{aligned}
$$


Therefore it is clear that the simple relationship (1.14) only holds in the case $N \in \mathbb{Z}$. The Matsubara sums to be defined next will in general be functions of several integer variables $N_{v}$. We will not consider in this paper the extension of our results to the case $N_{v}$ complex.

\section{Matsubara sums}

In this section we will introduce the class of sums $\mathfrak{M}$, whose elements we shall call Matsubara sums. These sums were considered for the first time by T. Matsubara [3] in his work on the statistical mechanics of quantum fields, where they appear in connection to the evaluation of so-called Feynman diagrams. In order to give a general definition of the class $\mathfrak{M}$ we need to make use of some graph-theoretical terminology.

Consider a connected graph formed by a set of $V$ points (also called vertices) and $I$ edges (also called lines or arcs). We will demand that each line joins two different vertices (that is, we exclude loops, i.e. lines that join a vertex to itself) and that each vertex be the endpoint of at least two different lines. Any pair of vertices can be joined by more than one line. In graph-theoretic language [4, [5], we are considering a $(V, I)$-multigraph such that the degree of each vertex is at least 2 .

We will restrict our attention to graphs of the type described, which we shall call Matsubara graphs.

Let $G$ be a Matsubara graph with $V$ vertices and $I$ lines. We choose for each line a definite orientation and assign to this oriented line a positive real number $q_{i}$ and an integer-valued summation variable $n_{i}$. We assign to each vertex $v$ an integer $N_{v}$ and the algebraic sum $T_{v}:=\sum_{i} s_{i}^{v} n_{i}$, where

$$
s_{i}^{v}= \begin{cases}+1 & \text { if the line } i \text { is oriented into vertex } v \\ -1 & \text { if the line } i \text { is oriented away from vertex } v \\ 0 & \text { if the line } i \text { is not incident on vertex } v\end{cases}
$$

The Matsubara sum $S_{G}$ associated to the graph $G$ is defined as

$$
S_{G}:=\sum_{n_{1}=-\infty}^{\infty} \sum_{n_{2}=-\infty}^{\infty} \cdots \sum_{n_{I}=-\infty}^{\infty} \frac{\delta_{G}\left(n_{1}, n_{2}, \ldots, n_{I} ;\left\{N_{v}\right\}\right)}{\left(n_{1}^{2}+q_{1}^{2}\right)\left(n_{2}^{2}+q_{2}^{2}\right) \cdots\left(n_{I}^{2}+q_{I}^{2}\right)} .
$$

Here $\delta_{G}\left(n_{1}, n_{2}, \ldots, n_{I} ;\left\{N_{v}\right\}\right)$ is a function valued in $\{0,1\}$ whose function is to impose a series of linear constraints among the summation variables $n_{i}$. It is given explicitly by

$$
\delta_{G}\left(n_{1}, n_{2}, \ldots, n_{I} ;\left\{N_{v}\right\}\right)=\prod_{v=1}^{V} \delta_{T_{v}, N_{v}},
$$

where $\delta_{m, n}$ is the Kronecker delta defined in (1.10) above. Whenever there is no possibility of confusion we will use the shorthand notation $\delta_{G}(n ; N)$ to denote the object $\delta_{G}\left(n_{1}, n_{2}, \ldots, n_{I} ;\left\{N_{v}\right\}\right)$.

Lemma 2.1. $S_{G}=0$, unless the integers $N_{v}$ satisfy the relation

$$
\sum_{v=1}^{V} N_{v}=0
$$



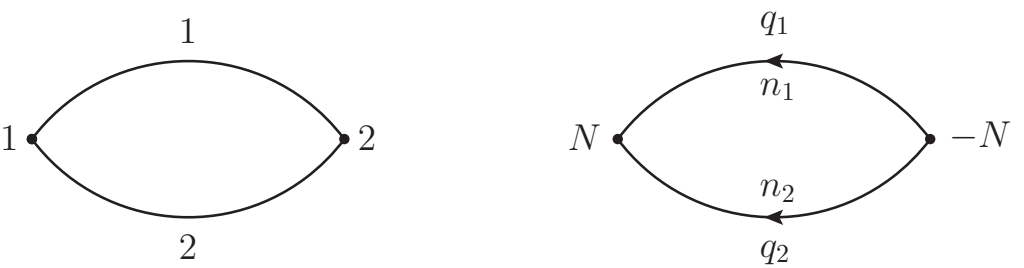

Figure 1. The graph $G_{2}$, with labeled vertices and lines (left) and its Matsubara sum variables (right).

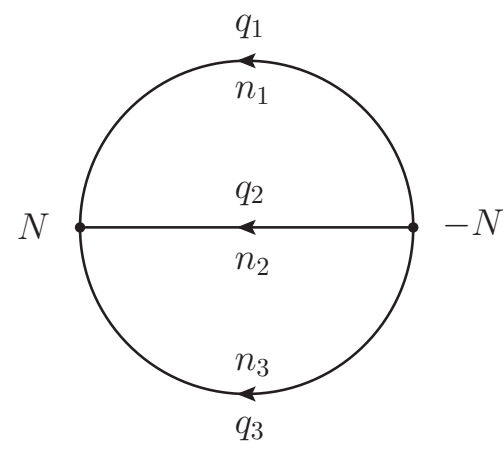

Figure 2. The graph $G_{3}$ with its Matsubara sum variables.

Proof. The $V$ equations $T_{v}=N_{v}$ have to be satisfied simultaneously for the sum $S_{G}$ not to vanish. Now, each summation variable $n_{i}$ (associated to line $i$ ) appears in only two of these equations (those corresponding to the vertices on which line $i$ is incident), once in the form $+n_{i}$ and once in the form $-n_{i}$. Therefore, $\sum_{v} T_{v} \equiv 0$ and

$$
\sum_{v} N_{v}=\sum_{v} T_{v}=0
$$

Hence, in order for the Matsubara sums to be considered not to vanish identically, we shall always assume that the condition (2.4) holds.

We now give a few examples of Matsubara sums.

Example 2.2. The simplest non-trivial example of a Matsubara sum corresponds to the $(2,2)$-graph $G_{2}$ containing 2 vertices joined together by two lines, with $N_{1}+$ $N_{2} \equiv 0$, shown in Figure 1. Setting $N_{1}=N$, the Matsubara sum for $G_{2}$ is simply the sum $S_{G_{2}}$ defined in (1.9) in the Introduction.

Example 2.3. A slightly more complicated example of a Matsubara sum is the one associated to the $(2,3)$-graph $G_{3}$, consisting of two vertices joined now by three lines, represented in Figure 2. Again we require $N_{2}=-N_{1} \equiv-N$, in which case the constraints at the vertices reduce to the single equation $n_{1}+n_{2}+n_{3}=N$. Thus,

$$
S_{G_{3}}\left(N, q_{1}, q_{2}, q_{3}\right)=\sum_{n_{1}} \sum_{n_{2}} \frac{1}{\left(n_{1}^{2}+q_{1}^{2}\right)\left(n_{2}^{2}+q_{2}^{2}\right)\left(\left(N-n_{1}-n_{2}\right)^{2}+q_{3}^{2}\right)},
$$




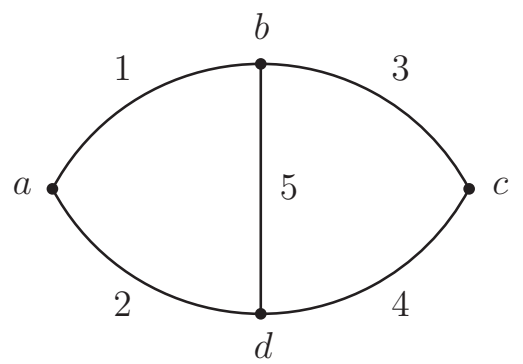

Figure 3 . The graph $G_{4}$, with labeled vertices and lines. The orientation of the lines, not shown, is described in the text.

where it is from now on understood that the summation variables $n_{i}$ run from $-\infty$ to $+\infty$.

As was the case for the sum $S_{G_{2}}$, it turns out that $S_{G_{3}}$ can also be generated in a simple way from the corresponding integral $I_{G_{3}}$, obtained by replacing the double sum by a double integral,

$$
\begin{aligned}
I_{G_{3}}\left(N, q_{1}, q_{2}, q_{3}\right) & =\int_{-\infty}^{\infty} d x_{1} \int_{-\infty}^{\infty} d x_{2} \frac{1}{\left(x_{1}^{2}+q_{1}^{2}\right)\left(x_{2}^{2}+q_{2}^{2}\right)\left(\left(N-x_{1}-x_{2}\right)^{2}+q_{3}^{2}\right)} \\
& =\frac{(2 \pi)^{2}}{2 q_{1} 2 q_{2} 2 q_{3}}\left[\frac{1}{N i+q_{1}+q_{2}+q_{3}}-\frac{1}{N i-q_{1}-q_{2}-q_{3}}\right] .
\end{aligned}
$$

In this case, after a lengthy evaluation, we find that

$$
S_{G_{3}}\left(N, q_{1}, q_{2}, q_{3}\right)=\hat{\mathcal{O}}_{G_{3}}^{\prime}\left(q_{1}, q_{2}, q_{3}\right) I_{G_{3}}\left(N, q_{1}, q_{2}, q_{3}\right),
$$

where $\hat{\mathcal{O}}_{G_{3}}^{\prime}$ is the operator,

$$
\begin{aligned}
\hat{\mathcal{O}}_{G_{3}}^{\prime}=1 & +\mathrm{n}_{\mathrm{b}}\left(q_{1}\right)\left(1-\hat{R}\left(q_{1}\right)\right)+\mathrm{n}_{\mathrm{b}}\left(q_{2}\right)\left(1-\hat{R}\left(q_{2}\right)\right)+\mathrm{n}_{\mathrm{b}}\left(q_{3}\right)\left(1-\hat{R}\left(q_{3}\right)\right) \\
& +\mathrm{n}_{\mathrm{b}}\left(q_{1}\right) \mathrm{n}_{\mathrm{b}}\left(q_{2}\right)\left(1-\hat{R}\left(q_{1}\right)\right)\left(1-\hat{R}\left(q_{2}\right)\right) \\
& +\mathrm{n}_{\mathrm{b}}\left(q_{1}\right) \mathrm{n}_{\mathrm{b}}\left(q_{3}\right)\left(1-\hat{R}\left(q_{1}\right)\right)\left(1-\hat{R}\left(q_{3}\right)\right) \\
& +\mathrm{n}_{\mathrm{b}}\left(q_{2}\right) \mathrm{n}_{\mathrm{b}}\left(q_{3}\right)\left(1-\hat{R}\left(q_{2}\right)\right)\left(1-\hat{R}\left(q_{3}\right)\right) .
\end{aligned}
$$

As in the case of $G_{2}$, it is direct to show that the operator $\left(1-\hat{R}_{1}\right)\left(1-\hat{R}_{2}\right)\left(1-\hat{R}_{3}\right)$ annihilates $I_{G_{3}}$ :

$$
\left(1-\hat{R}_{1}\right)\left(1-\hat{R}_{2}\right)\left(1-\hat{R}_{3}\right) I_{G_{3}} \equiv 0 .
$$

Therefore, the operator that generates the sum $S_{G_{3}}$ from the corresponding integral $I_{G_{3}}$ can be written in the multiplicative form,

$$
\hat{\mathcal{O}}_{G_{3}}=\prod_{i=1}^{3}\left[1+\mathrm{n}_{\mathrm{b} i}\left(1-\hat{R}_{i}\right)\right] .
$$

We will prove in the following sections that the types of relationship just described are generic for Matsubara sums.

Example 2.4. As a final, more intricate example we consider the Matsubara sum associated to the graph $G_{4}$, represented in Figure 3, $G_{4}$ is a $(4,5)$-graph. Its 
Matsubara sum will be a function of 3 integer variables, $N_{a}, N_{b}, N_{c}$, and 5 real positive variables, $q_{1}, \ldots, q_{5}$ :

$$
S_{G_{4}}:=\sum_{n_{1}, \ldots, n_{5}} \frac{\delta_{n_{1}+n_{2}, N_{a}} \delta_{n_{3}-n_{1}-n_{5}, N_{b}} \delta_{-n_{3}-n_{4}, N_{c}}}{\left(n_{1}^{2}+q_{1}^{2}\right)\left(n_{2}^{2}+q_{2}^{2}\right)\left(n_{3}^{2}+q_{3}^{2}\right)\left(n_{4}^{2}+q_{4}^{2}\right)\left(n_{5}^{2}+q_{5}^{2}\right)} .
$$

The orientation of the lines has been chosen such that line 5 flows from top to bottom and all the rest flow from right to left. Solving for the constraints imposed by the Kronecker deltas, we find that $S_{G_{4}}$ is equivalent to the double sum

$$
\begin{aligned}
& \sum_{n=-\infty}^{\infty} \sum_{m=-\infty}^{\infty}\left\{\frac{1}{\left(n^{2}+q_{1}^{2}\right)\left(m^{2}+q_{5}^{2}\right)}\right. \\
& \left.\times \frac{1}{\left(\left(N_{a}-n\right)^{2}+q_{2}^{2}\right)\left(\left(N_{b}+n+m\right)^{2}+q_{3}^{2}\right)\left(\left(N_{b}+N_{c}+n+m\right)^{2}+q_{4}^{2}\right)}\right\} .
\end{aligned}
$$

We shall present the explicit evaluation of this sum in section 6 .

\section{The MAIN THEOREMS}

Here we state the main theorems concerning the explicit evaluation of Matsubara sums. These results were first conjectured [6] and then proved [7] in the context of thermal quantum field theory. Our proofs of Theorems 3.1 and 3.4 and of Lemma 3.3 rely on the explicit evaluation of both the Matsubara sum and the Matsubara integral associated to a general graph $G$, which will be given in the next section.

Theorem 3.1. Let $G$ be a Matsubara graph. Let $\left\{q_{i}\right\}, i=1, \ldots, I$ be the set of positive real values associated to the lines of $G$ and $\left\{N_{v}\right\}, v=1, \ldots, V$ be the set of integer values associated to the vertices of $G$. We assume the condition $\sum_{v} N_{v}=0$ to hold. Then the Matsubara sum of $G, S_{G}\left(\left\{N_{v}\right\},\left\{q_{i}\right\}\right)$, can be explicitly evaluated as

$$
S_{G}\left(\left\{N_{v}\right\},\left\{q_{i}\right\}\right)=\hat{\mathcal{O}}_{G}\left(\left\{q_{i}\right\}\right) I_{G}\left(\left\{N_{v}\right\},\left\{q_{i}\right\}\right),
$$

where

$$
\hat{\mathcal{O}}_{G}\left(\left\{q_{i}\right\}\right)=\prod_{i=1}^{I}\left[1+\mathrm{n}_{\mathrm{b} i}\left(1-\hat{R}_{i}\right)\right],
$$

and $I_{G}\left(\left\{N_{v}\right\},\left\{q_{i}\right\}\right)$ is the Matsubara integral of $G$.

Upon expansion of the product, the operator $\hat{\mathcal{O}}_{G}$ can be seen to contain one or more terms that individually annihilate the Matsubara integral $I_{G}$, so that actually the operator $\hat{\mathcal{O}}_{G}$ can be made "smaller". To state this result we need the following graph-theoretical definition [5]:

Definition 3.2. A cutset of the (connected) graph $G$ is a set of lines whose removal from the graph results in a disconnected graph.

Lemma 3.3. Let $C$ be a cutset of the graph $G$. Then the operator

$$
\hat{\mathcal{A}}_{C}=\prod_{i \in C}\left(1-\hat{R}_{i}\right)
$$

annihilates the Matsubara integral $I_{G}$ associated to the graph $G$. 
Theorem 3.4. Let the conditions of Theorem 3.1 hold and let $L:=I-V+1$ be the number of independent cycles of the graph $G$. Then the relationship between the Matsubara sum $S_{G}\left(\left\{N_{v}\right\},\left\{q_{i}\right\}\right)$ and integral $I_{G}\left(\left\{N_{v}\right\},\left\{q_{i}\right\}\right)$ can alternatively be written as

$$
S_{G}\left(\left\{N_{v}\right\},\left\{q_{i}\right\}\right)=\hat{\mathcal{O}}_{G}^{\prime}\left(\left\{q_{i}\right\}\right) I_{G}\left(\left\{N_{v}\right\},\left\{q_{i}\right\}\right)
$$

where

$$
\begin{aligned}
\hat{\mathcal{O}}_{G}^{\prime}\left(\left\{q_{i}\right\}\right):=1 & +\sum_{i=1}^{I} \mathrm{n}_{\mathrm{b} i}\left(1-\hat{R}_{i}\right)+\sum_{\left\langle i_{1}, i_{2}\right\rangle}^{\prime} \mathrm{n}_{\mathrm{b} i_{1}} \mathrm{n}_{\mathrm{b} i_{2}}\left(1-\hat{R}_{i_{1}}\right)\left(1-\hat{R}_{i_{2}}\right) \\
& +\cdots+\sum_{\left\langle i_{1}, \ldots, i_{L}\right\rangle}^{\prime} \prod_{l=1}^{L} \mathrm{n}_{\mathrm{b} i_{l}}\left(1-\hat{R}_{i_{l}}\right) .
\end{aligned}
$$

Here the indices $i_{1}, i_{2}, \ldots$ run from 1 to $I$ (the number of lines of the graph $G$ ) and the symbol $\left\langle i_{1}, \ldots, i_{k}\right\rangle$ stands for an unordered $k$-tuple with no repeated indices, representing a particular set of lines. The prime on the summation symbols implies that the tuples that are cutsets of the graph $G$ are to be excluded from the sums.

Note that the operator $\hat{\mathcal{O}}_{G}^{\prime}\left(\left\{q_{i}\right\}\right)$ contains products of at most $L$ kernel factors $\mathrm{n}_{\mathrm{b}}\left(q_{i}\right)$, since for a graph with $L$ independent cycles the maximum number of lines that can be removed without disconnecting the graph is precisely $L 1$

\section{Explicit evaluations of $S_{G}$ And $I_{G}$}

We notice that the main building block of a Matsubara sum can be expressed as

$$
\begin{aligned}
\frac{1}{n^{2}+q^{2}} & =\frac{1}{2 q}\left[\frac{1}{i n+q}-\frac{1}{i n-q}\right] \\
& =\frac{1}{2 q}(1-\hat{R}(q)) \frac{1}{q-i n} .
\end{aligned}
$$

The representation above would allow us to trade the original quadratic denominators in the Matsubara sum (2.2) for linear denominators (in the summation variables $n_{i}$ ), and express $S_{G}$ in the form

$$
S_{G}=\prod_{k=1}^{I} \frac{1}{2 q_{k}}\left(1-\hat{R}_{k}\right) \sum_{n_{1}, \ldots, n_{I}} \frac{\delta_{G}\left(n_{1}, n_{2}, \ldots, n_{I} ;\left\{N_{v}\right\}\right)}{\left(q_{1}-i n_{1}\right)\left(q_{2}-i n_{2}\right) \cdots\left(q_{I}-i n_{I}\right)},
$$

were it not for the fact that the new sum does not converge in general. However, as we shall see, it is possible to regulate the sum in (4.2) in such a way that it is well defined.

The main results that will allow us to obtain a complete evaluation of the Matsubara sum $S_{G}$ were obtained by M. Gaudin [8] long ago, and will be reviewed in this section, adapted to the context of this paper. Gaudin showed that the summand in (4.2) admits a decomposition into partial fractions, which allows us to systematically eliminate the constraints imposed by the delta function $\delta_{G}(n ; N)$ and perform the sum explicitly.

\footnotetext{
${ }^{1}$ The number of independent cycles of a graph $G$ is called the cycle rank or cyclomatic number in graph theory and is given by $m(G)=I-V+1$ if $G$ is connected [5].
} 

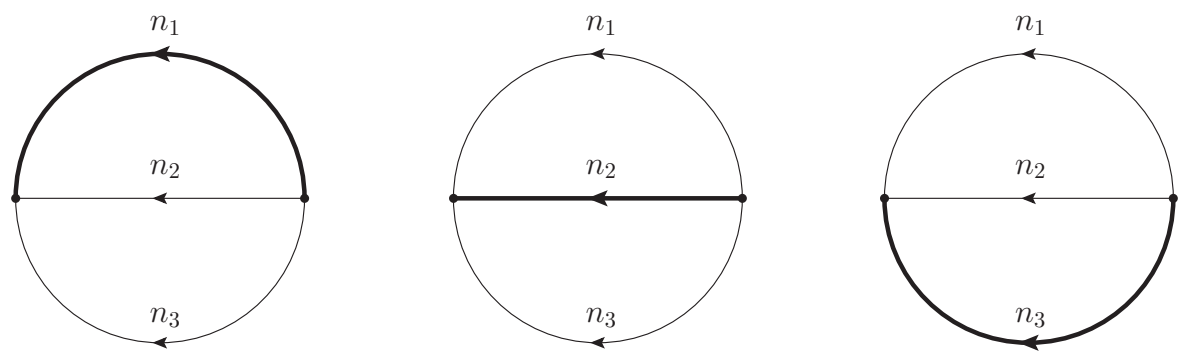

Figure 4 . The trees of the graph $G_{3}$, shown as dark lines.

The generalized Kronecker delta $\delta_{G}(n ; N)$ enforces $V-1$ independent linear relations satisfied by the summation variables $n_{i}$, also involving the vertex parameters $N_{v}$, which we shall write as

$$
R_{v}(N, n)=0, \quad \text { for } v=1, \ldots, V-1 .
$$

This system of linear equations allows us to solve for $V-1$ of the $I$ summation variables in terms of a set of $L=I-V+1$ independent ones. In general, there will be several distinct ways of choosing this set of independent summation variables. As shown by Gaudin [8, there is a one-to-one correspondence between the collection of all possible sets of independent summation variables and the set of all trees associated to the given (connected) graph $G$.

A tre 2 is a set of lines of $G$ joining all vertices and making a connected graph with no cycles. Every tree $\mathcal{T}$ will contain $V-1$ lines and its complement $\overline{\mathcal{T}}$ (the set of lines of $G$ which do not belong to $\mathcal{T}$ ) will have $L$ lines. The summation variables corresponding to the $L$ lines in $\overline{\mathcal{T}}$, denoted by $n_{l}$, will constitute a set of independent summation variables in terms of which the system (4.3) can be solved. The summation variables associated with the lines of the tree, $n_{j}$, with $j \in \mathcal{T}$, will be linear combinations of the independent summation variables and the vertex parameters,

$$
n_{j}=\Omega_{j}^{\mathcal{T}}\left(N, n_{l}\right), \quad j \in \mathcal{T}, l \in \overline{\mathcal{T}} .
$$

As a simple example, in Figure 4 we show the three possible trees for the $(2,3)$ graph $G_{3}$. In this case, each tree $\mathcal{T}$ is composed of a single line (heavy line), whose summation variable can be expressed, after solving for the constraint at one of the vertices imposed by the delta function, in terms of the two independent summation variables associated with the (thin) lines that do not belong to the tree (these are the lines in $\overline{\mathcal{T}}$ ) and the vertex parameter $N$. For instance, for the first tree we have $n_{1}=N-n_{2}-n_{3}$, etc.

Similarly, for the $(4,5)$-graph $G_{4}$ of Figure 3 there exist 8 trees, shown in Figure 5. The system of equations determined by the Kronecker deltas in the definition (2.11) of $S_{G_{4}}$ can be solved in terms of 8 different sets of independent variables, one for each of the 8 trees. For instance, the solution that led to the double sum (2.12), $n_{2}=N_{a}-n_{1}, n_{3}=N_{b}+n_{1}+n_{5}$ and $n_{4}=-N_{b}-N_{c}-n_{1}-n_{5}$, corresponds to the last tree in Figure 5 (the one in the bottom-right corner).

\footnotetext{
${ }^{2}$ More accurately, a spanning tree, in the graph-theoretical terminology.
} 

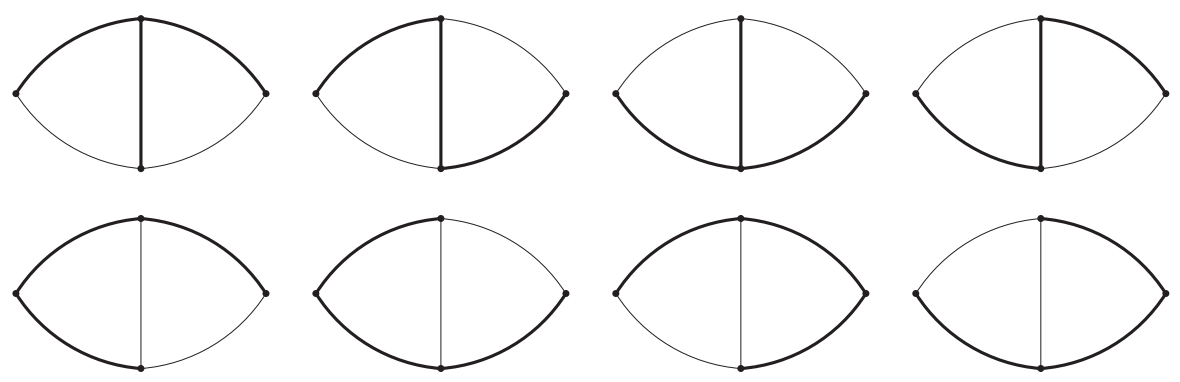

Figure 5 . The 8 trees of the graph $G_{4}$, represented by the dark lines.

Gaudin's main insight is the following identity for the rational function appearing in (4.2):

$$
\prod_{k=1}^{I} \frac{1}{q_{k}-i n_{k}} \delta_{G}(n ; N)=\sum_{\mathcal{T}} \prod_{j \in \mathcal{T}} \frac{1}{q_{j}-i \Omega_{j}^{\mathcal{T}}\left(N,-i q_{l}\right)} \prod_{l \in \mathcal{\mathcal { T }}} \frac{1}{q_{l}-i n_{l}} \delta_{G}(n ; N) .
$$

For instance, for the case of the Matsubara sum $S_{G_{3}}$, this identity takes the form

$$
\begin{aligned}
& \frac{\delta\left(N-n_{1}-n_{2}-n_{3}\right)}{\left(q_{1}-i n_{1}\right)\left(q_{2}-i n_{2}\right)\left(q_{3}-i n_{3}\right)}=\frac{\delta\left(N-n_{1}-n_{2}-n_{3}\right)}{\left(q_{1}+q_{2}+q_{3}-i N\right)} \\
& \times\left\{\frac{1}{\left(q_{2}-i n_{2}\right)\left(q_{3}-i n_{3}\right)}+\frac{1}{\left(q_{1}-i n_{1}\right)\left(q_{3}-i n_{3}\right)}+\frac{1}{\left(q_{1}-i n_{1}\right)\left(q_{2}-i n_{2}\right)}\right\} .
\end{aligned}
$$

We note that the identity (4.5) holds only in the presence of the constraint $\delta_{G}(n ; N)$ on the variables $n_{i}$. For instance, in the example above,

$$
\begin{aligned}
\frac{1}{\left(q_{2}-i n_{2}\right)\left(q_{3}-i n_{3}\right)}+\frac{1}{\left(q_{1}-i n_{1}\right)\left(q_{3}-i n_{3}\right)} & +\frac{1}{\left(q_{1}-i n_{1}\right)\left(q_{2}-i n_{2}\right)} \\
& =\frac{q_{1}+q_{2}+q_{3}-i\left(n_{1}+n_{2}+n_{3}\right)}{\left(q_{1}-i n_{1}\right)\left(q_{2}-i n_{2}\right)\left(q_{3}-i n_{3}\right)},
\end{aligned}
$$

so that the identity (4.6) holds only if the constraint $n_{1}+n_{2}+n_{3}=N$ is imposed.

Once we have applied Gaudin's identity (4.5) to the Matsubara sum (4.2), we proceed to perform the sum over the variables $n_{i}$. Since, for each tree, the summation variables $n_{j}, j \in \mathcal{T}$, appear now only in the constraint $\delta_{G}(n ; N)$, we have formally

$$
\sum_{n_{1}, \ldots, n_{I}} \prod_{l \in \overline{\mathcal{T}}} \frac{1}{q_{l}-i n_{l}} \delta_{G}(n ; N)=\sum_{n_{l}: l \in \overline{\mathcal{T}}} \prod_{l \in \mathcal{\mathcal { T }}} \frac{1}{q_{l}-i n_{l}}=\prod_{l \in \overline{\mathcal{T}}} \sum_{n_{l}} \frac{1}{q_{l}-i n_{l}} .
$$

Unfortunately, the sum

$$
\sum_{n=-\infty}^{\infty} \frac{1}{q-i n}
$$

diverges, and the result above does not make sense.

However, as shown in [8], it is possible to regulate the sum in such a way that all intermediate steps are mathematically sound. The basic idea is to associate to 
each line $i$ in the graph $G$ a real parameter $\tau_{i}$, which at the end will be taken to zero, and consider the sum

$$
S_{G}^{\tau}:=\sum_{n_{1}, \ldots, n_{I}} \frac{e^{i\left(n_{1} \tau_{1}+n_{2} \tau_{2}+\cdots+n_{I} \tau_{I}\right)}}{\left(n_{1}^{2}+q_{1}^{2}\right)\left(n_{2}^{2}+q_{2}^{2}\right) \cdots\left(n_{I}^{2}+q_{I}^{2}\right)} \delta_{G}\left(n_{1}, n_{2}, \ldots, n_{I} ;\left\{N_{v}\right\}\right),
$$

so that

$$
S_{G}=\lim _{\tau_{i} \rightarrow 0} S_{G}^{\tau}
$$

The subtle issues related to the interchange in the order of limits and summations are discussed at length in 8 . Now we have

$$
\sum_{n_{1}, \ldots, n_{I}} \prod_{l \in \mathcal{\mathcal { T }}} \frac{1}{q_{l}-i n_{l}} \delta_{G}(n ; N) e^{i\left(n_{1} \tau_{1}+n_{2} \tau_{2}+\cdots+n_{I} \tau_{I}\right)}=\prod_{l \in \mathcal{\mathcal { T }}} \sum_{n_{l}} \frac{e^{i n_{l} T_{l}}}{q_{l}-i n_{l}},
$$

where $T_{l}$ is a linear combination of the $\tau_{i}$, whose particular form depends on the tree $\mathcal{T}$ being considered. It can be shown that $T_{l}$ is the algebraic sum of the $\tau$-variables of the lines of the cycle $\mathcal{T} \cup\{l\}$ (formed by adding the line $l$ to the tree $\mathcal{T}$ ): $\tau_{k}$ will be preceded by a plus sign if the line $k$ has the same orientation as the line $l$, and by a minus sign otherwise.

The sum that we now need is given by

$$
\sum_{n} \frac{e^{i n T}}{q-i n}=2 \pi \varepsilon \mathrm{n}_{\mathrm{b}}(\varepsilon q) e^{T q}
$$

where $\varepsilon$ is the sign of $T(\varepsilon=+1$ if $T>0$ and $\varepsilon=-1$ if $T<0)$ and $\mathrm{n}_{\mathrm{b}}(z)$ is the kernel introduced in (1.5). Clearly, only $\varepsilon$ matters when the regulator $T$ is taken to zero.

The final result for the Matsubara sum for the graph $G$ is therefore

$$
S_{G}=\prod_{k=1}^{I} \frac{1}{2 q_{k}}\left(1-\hat{R}_{k}\right) \sum_{\mathcal{T}}\left(\prod_{j \in \mathcal{T}} \frac{1}{q_{j}-i \Omega_{j}^{\mathcal{T}}\left(N,-i q_{l}\right)} \prod_{l \in \mathcal{\mathcal { T }}} 2 \pi \varepsilon_{l} \mathrm{n}_{\mathrm{b}}\left(\varepsilon_{l} q_{l}\right)\right),
$$

where $\varepsilon_{l}$ is the sign of the variable $T_{l}$ associated to each line $l \in \overline{\mathcal{T}}$ in the process of regulation.

Consider now the (regulated) Matsubara integral associated to the graph $G$ :

$$
\begin{aligned}
& I_{G}^{\tau}:=\int \cdots \int d x_{1} d x_{2} \cdots d x_{I} \\
& \frac{e^{i\left(x_{1} \tau_{1}+x_{2} \tau_{2}+\cdots+x_{I} \tau_{I}\right)}}{\left(x_{1}^{2}+q_{1}^{2}\right)\left(x_{2}^{2}+q_{2}^{2}\right) \cdots\left(x_{I}^{2}+q_{I}^{2}\right)} \delta_{G}\left(x_{1}, x_{2}, \ldots, x_{I} ;\left\{N_{v}\right\}\right),
\end{aligned}
$$

so that

$$
I_{G}=\lim _{\tau_{i} \rightarrow 0} I_{G}^{\tau}
$$

Now $\delta_{G}$ stands for a product of Dirac delta functions, and the integrals over the $x$-variables run from $-\infty$ to $\infty$. 
All the algebraic manipulations given above for the sum $S_{G}$ hold here unchanged, leading us to

$$
\begin{aligned}
\int \cdots \int d x_{1} d x_{2} \cdots d x_{I} \prod_{l \in \mathcal{T}} \frac{1}{q_{l}-i x_{l}} \delta_{G}(x, N) e^{i\left(x_{1} \tau_{1}+x_{2} \tau_{2}+\cdots+x_{I} \tau_{I}\right)} & \\
& =\prod_{l \in \mathcal{T}} \int_{-\infty}^{\infty} \frac{e^{i x_{l} T_{l}}}{q_{l}-i x_{l}} d x_{l},
\end{aligned}
$$

where $T_{l}$ is the same linear combination of the $\tau_{i}$ as in the case of $S_{G}$.

The integral that we need now is given by

$$
\int_{-\infty}^{\infty} \frac{e^{i x T}}{q-i x} d x=-2 \pi \varepsilon \vartheta(-\varepsilon q) e^{T q},
$$

where $\varepsilon$ is the sign of $T$, and $\vartheta(q)$ is the Heaviside step function. Clearly, again only $\varepsilon$ matters when the regulator $T$ is taken to zero.

So,

$$
I_{G}=\prod_{k=1}^{I} \frac{1}{2 q_{k}}\left(1-\hat{R}_{k}\right) \sum_{\mathcal{T}}\left(\prod_{j \in \mathcal{T}} \frac{1}{q_{j}-i \Omega_{j}^{\mathcal{T}}\left(N,-i q_{l}\right)} \prod_{l \in \mathcal{\mathcal { T }}}-2 \pi \varepsilon_{l} \vartheta\left(-\varepsilon_{l} q_{l}\right)\right) .
$$

\section{The Relation Between $S_{G}$ And $I_{G}$}

In this section we use the explicit evaluations obtained in the previous section to prove the theorems stated in section 3 which provide an efficient way of computing the Matsubara sum $S_{G}$ in terms of the Matsubara integral $I_{G}$.

Lemma 5.1. The Matsubara integral can be written as

$$
I_{G}=\sum_{\mathcal{T}} I_{G}^{\mathcal{T}}
$$

where

$$
I_{G}^{\mathcal{T}}=(2 \pi)^{L} \prod_{k=1}^{I} \frac{1}{2 q_{k}} \prod_{j \in \mathcal{T}}\left(1-\hat{R}_{j}\right) \frac{1}{q_{j}-i \Omega_{j}^{\mathcal{T}}\left(N, i \varepsilon_{l} q_{l}\right)}
$$

is the contribution to $I_{G}$ associated to the tree $\mathcal{T}$.

Proof. Assuming $q_{l}>0$ we have

$$
\begin{aligned}
\left(1-\hat{R}_{l}\right)\left[\vartheta\left(-\varepsilon_{l} q_{l}\right) f\left(q_{l}\right)\right] & =\vartheta\left(-\varepsilon_{l} q_{l}\right) f\left(q_{l}\right)-\vartheta\left(\varepsilon_{l} q_{l}\right) f\left(-q_{l}\right) \\
& =-\varepsilon_{l} f\left(-\varepsilon_{l} q_{l}\right) .
\end{aligned}
$$

Therefore, for each tree $\mathcal{T}$, the action of the operators $\left(1-\hat{R}_{l}\right)$ with $l \in \overline{\mathcal{T}}$ can be performed explicitly in (4.18), to yield (5.2). Note that $\varepsilon_{l}^{2}=1$ and that there are $L=I-V+1$ factors $(2 \pi)$ (one for each line in $\overline{\mathcal{T}}$ ).

Lemma 5.2. For $q \in \mathbb{R}, q \neq 0$, the function $\mathrm{n}_{\mathrm{b}}(q)$ satisfies the identity

$$
\mathrm{n}_{\mathrm{b}}(q)=-\vartheta(-q)+\epsilon(q) \mathrm{n}_{\mathrm{b}}(|q|),
$$

where $\epsilon(q)$ is the sign of $q$.

Proof. For $q>0$, (5.3) is a trivial identity. For $q<0, \mathrm{n}_{\mathrm{b}}(q)=-1-\mathrm{n}_{\mathrm{b}}(-q)$ is an immediate consequence of the identity (1.7). 
Lemma 5.3. The Matsubara sum $S_{G}$ can be written as

$$
S_{G}=\sum_{\mathcal{T}} \prod_{l \in \mathcal{T}}\left[1+\mathrm{n}_{\mathrm{b} l}\left(1-\hat{R}_{l}\right)\right] I_{G}^{\mathcal{T}} .
$$

Proof. The identity (5.3) implies that

$$
\mathrm{n}_{\mathrm{b}}\left(\varepsilon_{l} q_{l}\right)=-\vartheta\left(-\varepsilon_{l} q_{l}\right)+\varepsilon_{l} \epsilon\left(q_{l}\right) \mathrm{n}_{\mathrm{b}}\left(\left|q_{l}\right|\right) .
$$

Then, as we computed already, for $q_{l}>0$ and an arbitrary function $f(q)$,

$$
\left(1-\hat{R}_{l}\right)\left[-\vartheta\left(-\varepsilon_{l} q_{l}\right) f\left(q_{l}\right)\right]=\varepsilon_{l} f\left(-\varepsilon_{l} q_{l}\right),
$$

whereas

$$
\begin{aligned}
\left(1-\hat{R}_{l}\right)\left[\varepsilon_{l} \epsilon\left(q_{l}\right) \mathrm{n}_{\mathrm{b}}\left(\left|q_{l}\right|\right) f\left(q_{l}\right)\right] & =\varepsilon_{l} \mathrm{n}_{\mathrm{b}}\left(\left|q_{l}\right|\right)\left[\epsilon\left(q_{l}\right) f\left(q_{l}\right)-\epsilon\left(-q_{l}\right) f\left(-q_{l}\right)\right] \\
& =\varepsilon_{l} \epsilon\left(q_{l}\right) \mathrm{n}_{\mathrm{b}}\left(\left|q_{l}\right|\right)\left(1+\hat{R}_{l}\right) f\left(q_{l}\right) .
\end{aligned}
$$

But

$$
\left(1+\hat{R}_{l}\right) f\left(-\varepsilon_{l} q_{l}\right)=\left(1+\hat{R}_{l}\right) f\left(q_{l}\right) .
$$

Therefore, for $q_{l}>0$,

$$
\left(1-\hat{R}_{l}\right) \mathrm{n}_{\mathrm{b}}\left(\varepsilon_{l} q_{l}\right) f\left(q_{l}\right)=\varepsilon_{l}\left[1+\mathrm{n}_{\mathrm{b} l}\left(1+\hat{R}_{l}\right)\right] f\left(-\varepsilon_{l} q_{l}\right),
$$

and hence

$$
\begin{aligned}
\prod_{l \in \mathcal{\mathcal { T }}}\left(1-\hat{R}_{l}\right)\left(\prod_{j \in \mathcal{T}} \frac{1}{q_{j}-i \Omega_{j}^{\mathcal{T}}\left(N,-i q_{l}\right)} \prod_{l \in \overline{\mathcal{T}}} 2 \pi \varepsilon_{l} \mathrm{n}_{\mathrm{b}}\left(\varepsilon_{l} q_{l}\right)\right) \\
=(2 \pi)^{L} \prod_{l \in \mathcal{\mathcal { T }}}\left[1+\mathrm{n}_{\mathrm{b} l}\left(1+\hat{R}_{l}\right)\right] \prod_{j \in \mathcal{T}} \frac{1}{q_{j}-i \Omega_{j}^{\mathcal{T}}\left(N, i \varepsilon_{l} q_{l}\right)} .
\end{aligned}
$$

The final result follows from the basic property,

$$
\frac{1}{q}(1+\hat{R}(q)) f(q)=(1-\hat{R}(q))\left[\frac{1}{q} f(q)\right] .
$$

Now we are finally in a position to prove the propositions of section 3

Proof of Theorem 3.1. We know from Lemma 5.3 that

$$
S_{G}=\sum_{\mathcal{T}} \prod_{l \in \mathcal{\mathcal { T }}}\left[1+\mathrm{n}_{\mathrm{b} l}\left(1-\hat{R}_{l}\right)\right] I_{G}^{\mathcal{T}} .
$$

But the identity

$$
(1-\hat{R}(q)) \frac{1}{q}(1-\hat{R}(q))=\frac{1}{q}(1+\hat{R}(q))(1-\hat{R}(q)) \equiv 0
$$

implies that all the operators $\left(1-\hat{R}_{j}\right)$, with $j \in \mathcal{T}$, annihilate each of the $I_{G}^{\mathcal{T}}$ defined in (5.2). Therefore we can extend the product indices in (5.6) from $l \in \overline{\mathcal{T}}$ to $i \in G$ :

$$
\sum_{\mathcal{T}} \prod_{l \in \overline{\mathcal{T}}}\left[1+\mathrm{n}_{\mathrm{b} l}\left(1-\hat{R}_{l}\right)\right] I_{G}^{\mathcal{T}}=\sum_{\mathcal{T}} \prod_{i \in G}\left[1+\mathrm{n}_{\mathrm{b} i}\left(1-\hat{R}_{i}\right)\right] I_{G}^{\mathcal{T}} .
$$


But now observe that the operator acting on $I_{G}^{\mathcal{T}}$ is $\mathcal{T}$-independent, so that we can transpose it with the sum over trees to get

$$
\begin{aligned}
S_{G} & =\prod_{i \in G}\left[1+\mathrm{n}_{\mathrm{b} i}\left(1-\hat{R}_{i}\right)\right] \sum_{\mathcal{T}} I_{G}^{\mathcal{T}} \\
& =\prod_{i \in G}\left[1+\mathrm{n}_{\mathrm{b} i}\left(1-\hat{R}_{i}\right)\right] I_{G},
\end{aligned}
$$

according to (5.1).

Proof of Lemma 3.3. Let $C$ be a cutset of the graph $G$ and let $\mathcal{T}$ be an arbitrary tree of $G$. The lines in $C$ cannot all belong to $\overline{\mathcal{T}}$ since then $C$ would not be a cutset (recall that $\mathcal{T}$ is a connected graph). Therefore, at least one of the lines in $C$ must belong to the tree $\mathcal{T}$, say $k$. But then the operator

$$
\hat{\mathcal{A}}_{C}=\prod_{i \in C}\left(1-\hat{R}_{i}\right)
$$

will contain the factor $\left(1-\hat{R}_{k}\right)$, which annihilates $I_{G}^{\mathcal{T}}$ in (5.2). Since this will be true for any tree, the result follows.

Proof of Theorem 3.4. The expansion of the product defining the operator $\hat{\mathcal{O}}_{G}$ generates an expression similar to (3.5), but where the sums run over all possible tuples of lines at each order. But according to Lemma 3.3 if a tuple $C$ is a cutset of $G$, then the corresponding term will contain the operator $\hat{\mathcal{A}}_{C}$, defined above, which annihilates the integral $I_{G}$. So the tuples corresponding to cutsets can safely be omitted from $\hat{\mathcal{O}}_{G}$. Finally, since a tree of $G$ has $V-1$ lines, then the maximum number of lines that we can remove without disconnecting the graph $G$ is $L=I-(V-1)$. So all tuples with more than $L$ lines will be cutsets, and hence the expansion of the product defining the operator $\hat{\mathcal{O}}_{G}$ ends at degree $L$.

\section{Example application: The calculation of $S_{G_{4}}$}

According to the results presented in this paper, we can compute the sum $S_{G_{4}}$ defined in (2.11) by first computing its associated integral $I_{G_{4}}$ and then acting on it with the operator $\hat{\mathcal{O}}_{G_{4}}^{\prime}$. According to Theorem 3.4, this operator is given by

$$
\begin{aligned}
\hat{\mathcal{O}}_{G_{4}}^{\prime}=1 & +\mathrm{n}_{\mathrm{b} 1}\left(1-\hat{R}_{1}\right)+\mathrm{n}_{\mathrm{b} 2}\left(1-\hat{R}_{2}\right) \\
& +\mathrm{n}_{\mathrm{b} 3}\left(1-\hat{R}_{3}\right)+\mathrm{n}_{\mathrm{b} 4}\left(1-\hat{R}_{4}\right)+\mathrm{n}_{\mathrm{b} 5}\left(1-\hat{R}_{5}\right) \\
& +\mathrm{n}_{\mathrm{b} 2} \mathrm{n}_{\mathrm{b} 4}\left(1-\hat{R}_{2}\right)\left(1-\hat{R}_{4}\right)+\mathrm{n}_{\mathrm{b} 2} \mathrm{n}_{\mathrm{b} 3}\left(1-\hat{R}_{2}\right)\left(1-\hat{R}_{3}\right) \\
& +\mathrm{n}_{\mathrm{b} 1} \mathrm{n}_{\mathrm{b} 3}\left(1-\hat{R}_{1}\right)\left(1-\hat{R}_{3}\right)+\mathrm{n}_{\mathrm{b} 1} \mathrm{n}_{\mathrm{b} 4}\left(1-\hat{R}_{1}\right)\left(1-\hat{R}_{4}\right) \\
& +\mathrm{n}_{\mathrm{b} 4} \mathrm{n}_{\mathrm{b} 5}\left(1-\hat{R}_{4}\right)\left(1-\hat{R}_{5}\right)+\mathrm{n}_{\mathrm{b} 3} \mathrm{n}_{\mathrm{b} 5}\left(1-\hat{R}_{3}\right)\left(1-\hat{R}_{5}\right) \\
& +\mathrm{n}_{\mathrm{b} 2} \mathrm{n}_{\mathrm{b} 5}\left(1-\hat{R}_{2}\right)\left(1-\hat{R}_{5}\right)+\mathrm{n}_{\mathrm{b} 1} \mathrm{n}_{\mathrm{b} 5}\left(1-\hat{R}_{1}\right)\left(1-\hat{R}_{5}\right),
\end{aligned}
$$

where, as before, $\mathrm{n}_{\mathrm{b} i} \equiv \mathrm{n}_{\mathrm{b}}\left(q_{i}\right)$ and $\hat{R}_{i} \equiv \hat{R}\left(q_{i}\right)$.

We note that the operator $\hat{\mathcal{O}}_{G_{4}}^{\prime}$ ends at degree 2 , since the removal of 3 or more lines from $G_{4}$ disconnects the graph. Moreover, each of the quadratic terms in (6.1) is associated with one of the trees of $G_{4}$ shown in Figure 5 . Note that there are no quadratic terms in (6.1) with index combinations $\langle 1,2\rangle$ and $\langle 3,4\rangle$, since these are cutsets of $G_{4}$ (see Figure 3 for the labeling). 
The integral $I_{G_{4}}$ associated to $G_{4}$ is given by (see (2.12)

$$
\begin{aligned}
I_{G_{4}}:=\int_{-\infty}^{\infty} & d x \int_{-\infty}^{\infty} d y\left\{\frac{1}{\left(x^{2}+q_{1}^{2}\right)\left(\left(N_{a}-x\right)^{2}+q_{2}^{2}\right)}\right. \\
& \left.\times \frac{1}{\left(\left(N_{b}+x+y\right)^{2}+q_{3}^{2}\right)\left(\left(N_{b}+N_{c}+x+y\right)^{2}+q_{4}^{2}\right)\left(y^{2}+q_{5}^{2}\right)}\right\} .
\end{aligned}
$$

This integral can be calculated following Gaudin's approach explained in section 4 or by directly performing the $x$ and $y$ integrations, one after the other, as we do now. Again, it is convenient to work with linear rather than quadratic denominators by expressing

$$
\frac{1}{x^{2}+q^{2}}=\frac{1}{2 q} \sum_{\varepsilon= \pm 1} \frac{\varepsilon}{i x+\varepsilon q}
$$

so that

$$
\begin{aligned}
& I_{G_{4}}=\frac{1}{2 q_{1} 2 q_{2} 2 q_{3} 2 q_{4} 2 q_{5}} \sum_{\varepsilon_{1}, \ldots \varepsilon_{5}= \pm 1} \int_{-\infty}^{\infty} d x\left\{\frac{\varepsilon_{1}}{\left(i x+\varepsilon_{1} q_{1}\right)} \frac{\varepsilon_{2}}{\left(i\left(N_{a}-x\right)+\varepsilon_{2} q_{2}\right)}\right. \\
& \left.\quad \times \int_{-\infty}^{\infty} d y \frac{\varepsilon_{3}}{\left(i\left(N_{b}+x+y\right)+\varepsilon_{3} q_{3}\right)} \frac{\varepsilon_{4}}{\left(i\left(N_{b}+N_{c}+x+y\right)+\varepsilon_{4} q_{4}\right)} \frac{\varepsilon_{5}}{\left(i y+\varepsilon_{5} q_{5}\right)}\right\} .
\end{aligned}
$$

Using Mathematica 7 to perform first the $y$-integral and then the $x$-integral we find

$$
\begin{aligned}
& \text { (6.4) } I_{G_{4}}=\frac{(2 \pi)^{2}}{2 q_{1} 2 q_{2} 2 q_{3} 2 q_{4} 2 q_{5}} \times \\
& {\left[\frac{1}{\left(i N_{a}+q_{1}+q_{2}\right)\left(i\left(N_{a}+N_{b}\right)+q_{2}+q_{3}+q_{5}\right)\left(i\left(N_{a}+N_{b}+N_{c}\right)+q_{2}+q_{4}+q_{5}\right)}\right.} \\
& +\frac{1}{\left(i N_{b}+q_{1}+q_{3}+q_{5}\right)\left(i\left(N_{a}+N_{b}\right)+q_{2}+q_{3}+q_{5}\right)\left(i\left(N_{a}+N_{b}+N_{c}\right)+q_{2}+q_{4}+q_{5}\right)} \\
& \quad-\frac{1}{\left(i N_{a}-q_{1}-q_{2}\right)\left(i N_{b}+q_{1}+q_{3}+q_{5}\right)\left(i\left(N_{b}+N_{c}\right)+q_{1}+q_{4}+q_{5}\right)} \\
& +\frac{1}{\left(i N_{b}+q_{1}+q_{3}+q_{5}\right)\left(i\left(N_{b}+N_{c}\right)+q_{1}+q_{4}+q_{5}\right)\left(i\left(N_{a}+N_{b}+N_{c}\right)+q_{2}+q_{4}+q_{5}\right)} \\
& -\frac{1}{\left(i N_{a}-q_{1}-q_{2}\right)\left(i N_{c}-q_{3}-q_{4}\right)\left(i\left(N_{a}+N_{b}+N_{c}\right)-q_{2}-q_{4}-q_{5}\right)} \\
& \quad+\frac{1}{\left(i N_{a}+q_{1}+q_{2}\right)\left(i N_{c}-q_{3}-q_{4}\right)\left(i\left(N_{b}+N_{c}\right)-q_{1}-q_{4}-q_{5}\right)} \\
& -\frac{1}{\left(i N_{c}-q_{3}-q_{4}\right)\left(i\left(N_{b}+N_{c}\right)-q_{1}-q_{4}-q_{5}\right)\left(i\left(N_{a}+N_{b}+N_{c}\right)-q_{2}-q_{4}-q_{5}\right)} \\
& \quad-\frac{1}{\left(i N_{a}+q_{1}+q_{2}\right)\left(i N_{c}-q_{3}-q_{4}\right)\left(i\left(N_{a}+N_{b}\right)+q_{2}+q_{3}+q_{5}\right)} \\
& \quad+\frac{1}{\left(i N_{c}-q_{3}-q_{4}\right)\left(i N_{b}+q_{1}+q_{3}+q_{5}\right)\left(i\left(N_{a}+N_{b}\right)+q_{2}+q_{3}+q_{5}\right)} \\
& +\frac{1}{\left(i N_{a}-q_{1}-q_{2}\right)\left(i N_{c}+q_{3}+q_{4}\right)\left(i\left(N_{a}+N_{b}\right)-q_{2}-q_{3}-q_{5}\right)}
\end{aligned}
$$




$$
\begin{gathered}
-\frac{1}{\left(i N_{a}+q_{1}+q_{2}\right)\left(i N_{c}+q_{3}+q_{4}\right)\left(i N_{b}-q_{1}-q_{3}-q_{5}\right)} \\
+\frac{1}{\left(i N_{c}+q_{3}+q_{4}\right)\left(i N_{b}-q_{1}-q_{3}-q_{5}\right)\left(i\left(N_{a}+N_{b}\right)-q_{2}-q_{3}-q_{5}\right)} \\
+\frac{1}{\left(i N_{a}+q_{1}+q_{2}\right)\left(i N_{c}+q_{3}+q_{4}\right)\left(i\left(N_{a}+N_{b}+N_{c}\right)+q_{2}+q_{4}+q_{5}\right)} \\
+\frac{1}{\left(i N_{a}-q_{1}-q_{2}\right)\left(i N_{c}+q_{3}+q_{4}\right)\left(i\left(N_{b}+N_{c}\right)+q_{1}+q_{4}+q_{5}\right)} \\
-\frac{1}{\left(i N_{c}+q_{3}+q_{4}\right)\left(i\left(N_{b}+N_{c}\right)+q_{1}+q_{4}+q_{5}\right)\left(i\left(N_{a}+N_{b}+N_{c}\right)+q_{2}+q_{4}+q_{5}\right)} \\
-\frac{1}{\left(i N_{a}-q_{1}-q_{2}\right)\left(i\left(N_{a}+N_{b}\right)-q_{2}-q_{3}-q_{5}\right)\left(i\left(N_{a}+N_{b}+N_{c}\right)-q_{2}-q_{4}-q_{5}\right)} \\
-\frac{1}{\left(i N_{b}-q_{1}-q_{3}-q_{5}\right)\left(i\left(N_{a}+N_{b}\right)-q_{2}-q_{3}-q_{5}\right)\left(i\left(N_{a}+N_{b}+N_{c}\right)-q_{2}-q_{4}-q_{5}\right)} \\
+\frac{1}{\left(i N_{a}+q_{1}+q_{2}\right)\left(i N_{b}-q_{1}-q_{3}-q_{5}\right)\left(i\left(N_{b}+N_{c}\right)-q_{1}-q_{4}-q_{5}\right)} \\
\left.-\frac{1}{\left(i N_{b}-q_{1}-q_{3}-q_{5}\right)\left(i\left(N_{b}+N_{c}\right)-q_{1}-q_{4}-q_{5}\right)\left(i\left(N_{a}+N_{b}+N_{c}\right)-q_{2}-q_{4}-q_{5}\right)}\right] .
\end{gathered}
$$

The explicit evaluation of sum $S_{G_{4}}$ can be obtained from the application of the operator $\hat{\mathcal{O}}_{G_{4}}^{\prime}$ given by (6.1) to the result (6.4) for the integral $I_{G_{4}}$. The resulting expression would fill several pages of this journal, but it can be easily generated by a symbolic manipulation program such as Mathematica.

\section{ACKNOWLEDGMENTS}

The author would like to thank the hospitality of the Center for Astronomy and Particle Theory at the University of Nottingham, where this work was written, and the financial support of Fondecyt, under grant 1070505. The diagrams presented in this paper were produced with JaxoDraw 2.0 [.

\section{REFERENCES}

[1] A. Erdélyi, W. Magnus, F. Oberhettinger, and F. Tricomi (Eds.), Higher Transcendental Functions, vol. 1, McGraw-Hill, 1953. MR0058756 (15:419i)

[2] E. T. Whittaker and G. N. Watson, A Course of Modern Analysis, Cambridge University Press, reprint of the Fourth (1927) Edition, 1996. MR 1424469 (97k:01072)

[3] T. Matsubara, A New Approach to Quantum-Statistical Mechanics. Progr. Theoret. Phys. 14, 351-378 (1955). MR0075107(17:695d)

[4] C. Berge, Graphs, North Holland, 1991.

[5] F. Harary, Graph Theory, Addison-Wesley, 1969. MR0256911 (41:1566)

[6] O. Espinosa and E. Stockmeyer, Operator representation for Matsubara sums. Phys. Rev. D69, 065004 (2004). MR2093145 (2005f:81158)

[7] O. Espinosa, Thermal operator representation for Matsubara sums. Phys. Rev. D71, 065009 (2005).

[8] M. Gaudin, Méthode d'intégration sur les variables d'énergie dans les graphes de la théorie des perturbations. Nuovo Cimento 38, 844-871 (1965). MR0198889 (33:7042)

[9] D. Binosi and L. Theußl, Comp. Phys. Comm. 161, 76 (2004).

Departamento de Física, Universidad Téc. Federico Santa María, Valparaíso, Chile E-mail address: olivier.espinosa@usm.cl 